\title{
A rare case report on atypical odontalgia with psychological implications
}

\author{
Pandya $\mathrm{D}^{1}$, Anil Kumar Nagarajappa ${ }^{2}$ \\ ${ }^{1}$ Dr. Divya Pandya BDS, Postgraduate Student, ${ }^{2}$ Dr. Anil Kumar Nagarajappa, MDS, Professor and Head, Both are \\ affiliated with Department of Oral Medicine and Radiology, Hitkarini Dental College and Hospital, Jabalpur, Madhya \\ Pradesh, India.
}

Address for Correspondence: Dr. Divya Pandya, M.P, F-2 Atul Vihar II S.B.I. Colony, Near Hathital Railway Crossing, Gorakhpur, Jabalpur, M.P, Email Id: divyapandya854@gmail.com

\begin{abstract}
Psychosomatic or somatoform disorders are among the most common psychiatric disorders found in general practice. A psychosomatic disorder involves both body and mind. These diseases have physical symptoms originating from mental or emotional causes. Most common ones are stress, anxiety and depression. A wide spectrum of psychiatric disorders may influence the orofacial region, where unfortunately they remain unrecognized due to limited nature of their presenting features. We here present a case report of atypical odontalgia in an elderly male secondary to underlying psychological distress.
\end{abstract}

Key words: Atypical, Liaison psychiatry, Oral diseases, Psychosomatic, Stress, Substance abuse

\section{Introduction}

Oral health is an integral part of overall health [1]. Mouth is directly or symbolically related to major human instincts and passion. The oral mucosa is highly reactive to psychological influences. Psychological disorders are defined as disorders characterized by psychological changes originating atleast in part, from emotional factors $[2,3]$. There is evidence that patients suffering from mental illness are found to be more vulnerable to dental neglect and poor oral health. Psychiatric disorders affect the overall behavior of a person, impair level of functioning and alter perception towards oral health. Alternatively, oral symptoms could be the first or only manifestation of a mental health problem like atypical facial pain, burning mouth syndrome, lichen planus, self inflicted injury etc. Dentists spend a considerable amount of time treating patients presenting with either psychiatric disorders like depression and anxiety or with physical manifestations of underlying emotional disturbances [1]. Psychosomatic disorders may be due to several biochemical alterations involving neurotransmitters in

Manuscript received: $02^{\text {nd }}$ Jan 2016

Reviewed: $13^{\text {th }}$ Jan 2016

Author Corrected: $24^{\text {th }}$ Jan 2016

Accepted for Publication: $03^{\text {rd }}$ Feb 2016 brain, incomplete connections with in oral region and undefined complaints due to cognitive processes in higher brain centers [4]. Increasing attention needs to be focused to identify and appropriately treat somatoform

disorders constituting one-third to one-half of referrals to any liaison psychiatry service. Recognizable psychopathology has been seen in up to $30 \%$ of patients attending dental clinics and this often goes undetected and hence treated. Dental specialists often come across patients presenting with complaints of pain, abnormal sensations, movement and salivation which are a manifestation of underlying emotional disturbance and not due to a clearly identifiable physical cause. Thus there is a need for early and appropriate recognition of such emotional distress that would benefit both the individual and the health service $[1,5]$.

\section{Case Report}

A 55 year old male patient reported to department of Oral Medicine and Radiology with a chief complaint of pain in upper front tooth region of jaw since 1 year. History revealed that pain was gradual in onset and 
progression, severe in intensity, intermittent, throbbing nature, exacerbated on lying down at night and during bath and was not associated with any swelling, discharge, bleeding or referred pain. There was an uneventful history of extraction of upper front tooth 1.5 years back under local anesthesia. Medical history was non contributory. Because of patient's confusing and irrelevant answers and behavior in clinic we enquired his family members to clear our suspicion of an underlying psychosomatic disorder. Thorough personal, family and habit history was recorded after interrogating the patient's wife and son which revealed that patient was a college drop out and a government ordinance factory employee with a good family background. Patient had very high hopes and expectations from his career and failure to do so lead him to chronic consumption of ganja (marijuana), alcohol, tobacco and smoking bidi all day since 30 years. As a result there were physical and psychotic manifestations of these abusive habits in the form of dilated pupil, tachycardia, excessive sweating, disorientation with history of hallucinations, hydrophobia, delusion of infidelity, fights with wife, phobia towards particular weather, peculiar sounds and chess board. On intraoral examination only 3 teeth were evident in maxillary arch with no evidence of any tooth, root stump, swelling, sinus opening or bony pathology in area of chief complaint (Figure 1) and there was completely edentulous lower arch. Hospital anxiety and depression rating scale was employed and a score of 19 indicating severe depression was obtained.

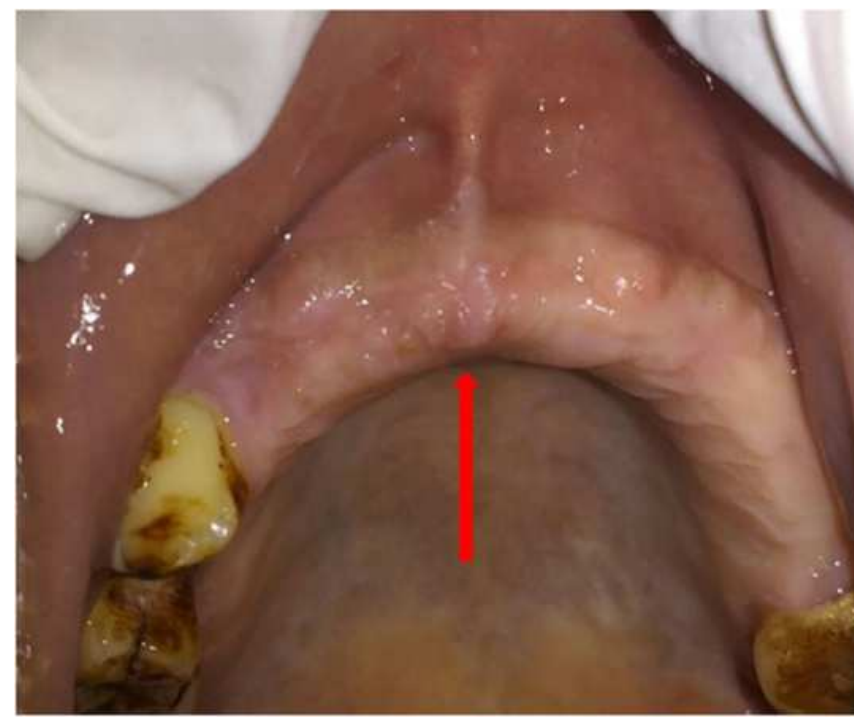

Figure-1: Edentulous area in maxillary front tooth region without evidence of any tooth or pathology
On the basis of significant personal, family and habit history of patient, clinical and radiological examination a diagnosis of Atypical odontalgia secondary to substance abuse was given. Patient was immediately counseled and referred to a psychiatrist and rehabilitation center after a placebo treatment. An introral periapical radiograph of the region was made which also revealed no pathology (Figure 2).

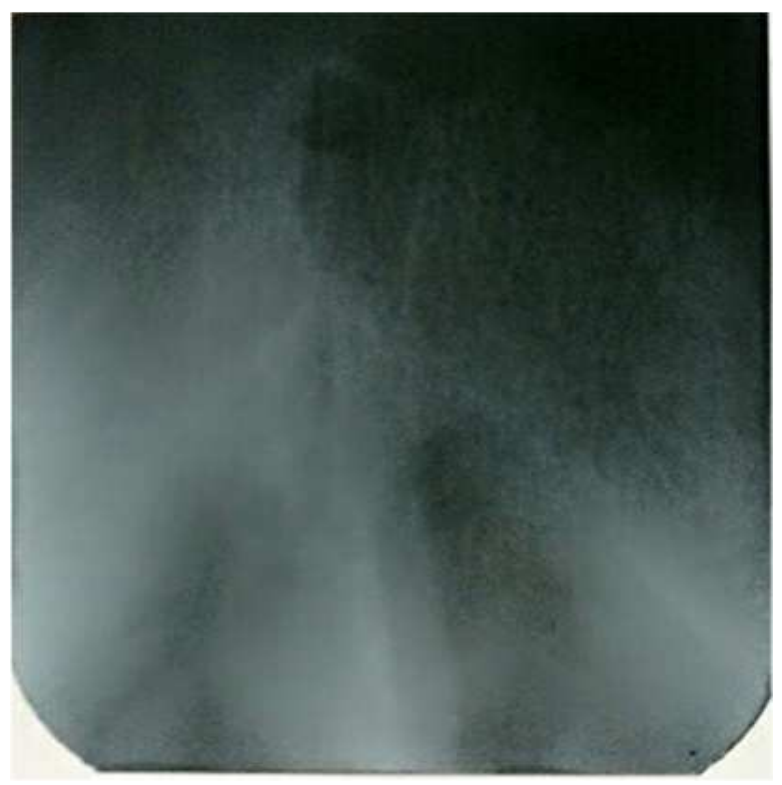

Figure-2: Intraoral periapical radiograph of maxillary front tooth region without evidence of any pathology

\section{Discussion}

Given the high prevalence of mental disorders in general population, dentists frequently treat patients who have noticeable abnormal behavior as well as with psychiatric disorders that are not easily identified or obvious. There is a need for dentists to be aware of patient vulnerability factors and psychological problems due to the possible negative effects of psychological distress and critical incidents, and their consequences for both symptom presentation and dental treatment planning. Dentists usually do not ask questions about one's psychological health, except that of dental anxiety. Dentists may avoid this aspect of a patient's health history as they feel that they are not trained to approach this topic or because they do not see its relevance to dental symptoms [6].

Biomarkers frequently used to assess stress level are nervous system markers such as adrenaline, nor adrenaline and dopamine. Endocrine system markers such as corticocoids in blood, urine and saliva and 
immune system markers like $\mathrm{T}$ and $\mathrm{B}$ cells, natural killer cells, interleukins, interferon and tumor necrosis factor $[3,7]$.

Psychiatric disorders most commonly encountered in dental clinics (ICD-10) include mood, anxiety, somatoform, eating disorders and substance abuse as with our case [6]. Diagnostic and Statically Manual, Mental Disorder (DSM-IV) proposed a working type classification for oral psychosomatic disorders in which our case fits in pain related disorders category[2,4]. Atypical odontalgia (AO) is probably one of the most frustrating conditions that challenge dental clinicians. It presents as tooth pain or pain in a site where a tooth was extracted, in absence of clinical and radiographic evidence of tooth pathology as with our parent case. The cause and pathophysiology of AO remains enigma. Several theories of de-afferentiation, vascular or neurovascular and psychological mechanisms have been proposed $[8,9]$.

Management of such patients requires consultationliaison psychiatric units with behavioral relaxation techniques, yoga or medidation, hypnosis, biofeedback, cognitive behavioral therapy and pharmacological management with antianxiety, antidepressants and sedative-hypnotics and antipsychotic drugs $[2,3,6,10]$.

\section{Conclusion}

Given the prevalence and impact of unrecognized and untreated psychiatric disorders in patients presenting in dental practice, there is a need for a service to address this unmet need. This would directly provide a framework for psychiatric dental liaison and indirectly lead to better understanding of psychiatric disorders by dental specialists thus in turn will lead to early identification and referral to such a service if one exists.

\section{Funding: Nil}

Conflict of interest: None.

Permission of IRB: Yes
1. Tomar B, Bhatia NK, Kumar P, Bhatia MS, Shah RJ. The psychiatric and dental interrelationship. Delhi Psychiatry Journal. Apr 2011; 14(1):138-42

2. Patil PB, Savalagi AG. Psychosomatic disorders of the oral cavity - A review. AJOMR. 2015; 2(2):96-102

3. Kandagal S, Shenai P, Chatra L, Ronad YAA, Kumar M. Effect of stress on oral mucosa. BBR. 2011/2012;1(1):13-16

4. Shamim T. The psychosomatic disorders pertaining to dental practice with revised working type classification. KJP. Jan 2014; 27(1):16-22

5. Richter I, Vidas I, Turcinovic P. Relationship of psychological characteristics and oral diseases with possible psychosomatic aetiology. Acta Stomatol Croat. 2003;37(1):35-9

6. Bathla M, Chandna S, Mehta DS, Grover HS. Dentistry and psychiatry: It's time to bridge the gap. Delhi Psychiatry Journal. Apr 2015; 18(1):20-4

7. Maheswari TNU, Gnanasundaram N. Stress related oral diseases- A research study. IJPBS. Jul-Sep 2010; 1(3):1-10

8. Melis M, Lobo SL, Ceneviz C, Zawawi K, AlBadawai E, Maloney G et al. Atypical odontalgia: A review of the literature. Headache. 2003; 43(10):106074

9. Issrani R, Prabhu N, Mathur S. Atypical facial pain and atypical odontalgia: A concise review. IJCDMR. 2015:1-4

10. Jain K, Mehendiratta M, Kardam P, Yadav J, Jindal DG. Cross talk between mind and oral cavity: An insight into pathogenesis, classification, presentation and management of oral psychosomatic disorders. INDJ. 2016; 5(2):1-12

\section{References}

\section{How to cite this article?}

Pandya D, Anil Kumar Nagarajappa. A rare case report on atypical odontalgia with psychological implications. Int $J$ Med Res Rev 2016;4(2):261-263. doi: 10.17511/ijmrr.2016.i02.001. 